\title{
Usefulness of antibody index assessment in cerebrospinal fluid from patients negative for total-IgG oligoclonal bands
}

\author{
Sven Jarius ${ }^{*}$, Peter Eichhorn², Brigitte Wildemann ${ }^{1}$ and Manfred Wick ${ }^{2}$
}

\begin{abstract}
Background: Testing for cerebrospinal fluid (CSF)-restricted oligoclonal bands (OCB) by isoelectric focusing is used to detect intrathecally produced total IgG. By contrast, antibody indices (Al) are assessed to test for intrathecally produced antigen-specific lgG. A number of previous cases reports have suggested that Al testing might be more sensitive than OCB testing in detecting intrathecal lgG synthesis.
\end{abstract}

Findings: Here we report on 21 patients with positive Al for either herpes simplex virus, varicella zoster virus, cytomegalovirus, measles virus, rubella virus, or Borrelia burgdorferi in the absence of total-IgG OCB and, accordingly, in the presence of a normal total-IgG CSF/serum ratio.

Conclusion: Our findings indicate that Al testing should not generally be omitted in OCB-negative patients and provide a rationale for systematic and prospective studies on the comparative sensitivity and specificity of Al and total-IgG OCB testing in infectious and other diseases of the CNS.

Keywords: Intrathecal IgG synthesis, Antibody index, Cerebrospinal fluid, Oligoclonal bands, Herpes simplex virus, Cytomegalovirus, Varicella zoster virus, Measles virus, Rubella virus, Borrelia burgdorferi, Multiple sclerosis, MRZ reaction

\section{Introduction}

Testing for cerebrospinal fluid (CSF)-restricted total IgG oligoclonal bands (OCB) by isoelectric focusing is a highly sensitive method for detecting intrathecal IgG synthesis, but does not take into account antibody specificity. By contrast, antibody indices (AI) are assessed to test for antigen-specific intrathecal IgG synthesis [1]. It is unclear whether AI assessment is useful in OCB negative patients. Currently, clinicians often do not test for virus- or bacterium-specific AIs on cost grounds if evidence for intrathecal total IgG synthesis as detected by OCB testing is missing.

Back in 1992, however, Felgenhauer and Reiber reported a positive varicella zoster virus (VZV)-specific AI in 6 total-IgG OCB-negative patients with VZV ganglionitis as well as in 4 total-IgG OCB-negative patients with VZV

\footnotetext{
* Correspondence: sven.jarius@med.uni-heidelberg.de

'Division of Molecular Neuroimmunology, Department of Neurology, University of Heidelberg, Im Neuenheimer Feld 400, 69120 Heidelberg, Germany

Full list of author information is available at the end of the article
}

meningitis, suggesting that $\mathrm{AI}$ calculation might be more sensitive than total-IgG OCB testing [2]. Here, we report on 21 total-IgG OCB-negative patients with positive AI results for either herpes simplex virus (HSV), VZV, cytomegalovirus $(\mathrm{CMV})$, measles virus $(\mathrm{MV})$, rubella virus (RV), or Borrelia burgdorferi (Bb), thereby providing independent and corroborative evidence for Felgenhauer and Reiber's hypothesis (see Table 1 for details).

\section{Methods and results}

Testing for OCB was performed by a CSF laboratory with long-standing expertise in the field that takes part in the official German external quality assessment organized by INSTAND e.V. twice a year. OCB were determined by isoelectric focusing followed by anti-IgG immunofixation (Sebia, Fulda, Germany). Serum and CSF levels of antibodies to HSV, VZV, CMV, MV, RV, and $\mathrm{Bb}$ were assessed using commercially available enzyme linked immunosorbent assays (Enzygnost, Siemes Healthcare, Eschborn, Germany). Total-IgG and total albumin concentrations in CSF and serum were

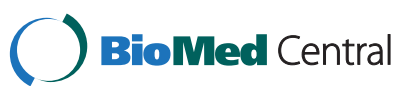


Table 1 Patient data and antibody indices to six viral antigens in CSF samples negative for total-IgG oligoclonal bands

\begin{tabular}{|c|c|c|c|c|c|c|c|c|c|c|c|}
\hline No. & Age & Sex & OCBs & $\begin{array}{l}\mathrm{Al} \\
\mathrm{Bb}\end{array}$ & $\begin{array}{l}\text { Al } \\
\text { HSV }\end{array}$ & $\begin{array}{l}\text { Al } \\
\text { VZV }\end{array}$ & $\begin{array}{l}\text { Al } \\
\text { CMV }\end{array}$ & $\begin{array}{l}\text { Al } \\
\text { MV }\end{array}$ & $\begin{array}{l}\mathrm{Al} \\
\mathrm{RV}\end{array}$ & $\begin{array}{l}\text { CSF } \\
\text { WCC }\end{array}$ & Suspected diagnosis and indicated by the sender \\
\hline$\# 1 \mathrm{~A}$ & 32 & M & NEG/NEG & Not det. & 0.72 & 0.74 & Not det. & 0.69 & 0.77 & 1557 & CSF PCR positive HSV encephalitis \\
\hline$\# 1 B$ & 32 & M & NEG/NEG & Not det. & 43.40 & 1.24 & Not det. & ND & ND & 1456 & CSF PCR positive HSV encephalitis, follow-up 19 days later \\
\hline$\# 1 \mathrm{C}$ & 32 & M & NEG/NEG & ND & 53.40 & $16.30^{\#}$ & ND & ND & ND & 152 & CSF PCR positive HSV encephalitis, follow-up a further 3 days later \\
\hline$\# 2 \mathrm{~A}$ & 33 & M & NEG/NEG & Not det. & 2.02 & 1.54 & ND & ND & 0.83 & No data & Blistering rash $\mathrm{V} 2$, peripheral facialis paresis \\
\hline$\# 2 \mathrm{~B}$ & 33 & M & NEG/NEG & ND & 1.94 & 1.39 & ND & ND & ND & 19 & Blistering rash V2, peripheral facialis paresis, follow-up 8 days later \\
\hline \#3 & 30 & M & NEG/NEG & ND & 2.43 & 1.12 & ND & 0.85 & 1.10 & 2 & No data available \\
\hline \#4 & 29 & F & NEG/NEG & Not det. & 2.12 & 0.99 & 1.30 & ND & 0.94 & 3 & No data available \\
\hline$\# 5 \mathrm{~A}$ & 34 & $\mathrm{~F}$ & NEG/NEG & Not det. & ND & ND & ND & ND & ND & No data & VZV meningoencephalitis \\
\hline \#5B & 34 & $\mathrm{~F}$ & NEG/NEG & ND & Not det. & 3.99 & Not det. & ND & ND & 360 & VZV meningoencephalitis, follow-up 5 days later \\
\hline \#6 & 60 & $\mathrm{~F}$ & NEG/NEG & Not det. & 1.20 & 2.40 & 0.86 & ND & ND & 2 & "FK506-associated leukoencephalopathy", grand mal \\
\hline \#7 & 51 & M & NEG/NEG & 1.26 & 1.11 & 2.42 & 1.03 & 0.90 & 1.03 & 1 & "Fatigue and apathy" \\
\hline \#8 & 42 & M & NEG/NEG & Not det. & 1.31 & 3.26 & ND & 1.03 & 1.32 & 3 & "Possible multiple sclerosis" \\
\hline \#9 & 74 & $\mathrm{~F}$ & NEG/NEG & ND & 1.43 & 2.30 & Not det. & ND & 0.81 & 2 & No data available \\
\hline$\# 10$ & 50 & $\mathrm{~F}$ & NEG/NEG & ND & Not det. & 2.00 & ND & 0.85 & 0.67 & No data & No data available \\
\hline$\# 11$ & 71 & $\mathrm{~F}$ & POS/POS* & 20.90 & 1.45 & 1.32 & Not det. & 0.97 & 0.91 & 4 & Neuroborreliosis, encephalitis \\
\hline$\# 12 \mathrm{~A}$ & 34 & $\mathrm{~F}$ & NEG/NEG & 2.60 & 0.91 & 0.80 & Not det. & 0.70 & 0.80 & 82 & Neuroborreliosis with abducens and facial nerve paresis \\
\hline$\# 12 B$ & 34 & $\mathrm{~F}$ & NEG/NEG & 1.70 & 0.91 & 0.95 & Not det. & 1.15 & 0.83 & 277 & Confirmatory follow-up sample obtained 5 days later \\
\hline$\# 13$ & 44 & M & NEG/NEG & 10.60 & 0.90 & 1.00 & 0.88 & 1.5 & ND & No data & "Aseptic Meningitis" \\
\hline$\# 14$ & 84 & M & NEG/NEG & 5.31 & 1.02 & 0.99 & 0.85 & 1.02 & 1.04 & 2 & No data available \\
\hline \#15 & 34 & F & NEG/NEG & 4.79 & Not det. & 0.99 & Not det. & 0.92 & ND & 1 & No data available \\
\hline$\# 16$ & 57 & M & NEG/NEG & 3.30 & 0.96 & 0.88 & 1.20 & 0.76 & 0.75 & 1 & No data available \\
\hline \#17 & 21 & M & NEG/NEG & 2.50 & Not det. & 0.85 & Not det. & ND & 0.85 & 1 & No data available \\
\hline$\# 18$ & 38 & $\mathrm{~F}$ & NEG/NEG & ND & 1.67 & 1.00 & 2.40 & 0.44 & 0.94 & No data & "Encephalitis" \\
\hline$\# 19$ & 40 & $\mathrm{~F}$ & NEG/NEG & Not det. & Not det. & 16.20 & 1.43 & 0.72 & 0.90 & No data & No data available \\
\hline$\# 20$ & 25 & F & NEG/NEG & Not det. & 0.92 & 1.00 & Not det. & ND & 2.10 & 1 & "Clinically possible multiple sclerosis (Poser)" \\
\hline$\# 21$ & 60 & M & NEG/NEG & ND & 0.85 & 0.87 & Not det. & 2.80 & 0.92 & No data & No data available \\
\hline
\end{tabular}

$\mathrm{Al}=$ antibody index; $\mathrm{Bb}=$ Borrelia burgdorferi; $\mathrm{CMV}=$ cytomegalovirus; $\mathrm{HSV}=$ herpes simplex virus; $\mathrm{MV}=$ measles virus; $\mathrm{NEG} / \mathrm{NEG}=$ negative in $\mathrm{CSF} / \mathrm{negative}$ in serum; not det. = not detectable; $\mathrm{ND}=$ not done; $\mathrm{OCBs}=$ oligoclonal bands; RV = rubella virus; VZV= varicella zoster virus. * Mirror pattern (so-called "pattern 4 " according to an international consensus on OCB diagnostics) in the absence of CSF-restricted lgG bands. " Cross-reactivity between HSV and VZV as a result of epitope spreading (see reference [2]).

determined nephelometrically (BN ProSpec, Dade Behring, Germany). Intrathecal synthesis of IgG to HSV, $\mathrm{VZV}, \mathrm{CMV}, \mathrm{MV}, \mathrm{RV}$, and $\mathrm{Bb}$ was determined by calculating the respective antibody indices $(\mathrm{AI}): \mathrm{AI}=\mathrm{QIgG}[\mathrm{spec}] /$ QIgG[total], if QIgG[total]<Qlim, and AI=QIgG[spec]/ Qlim, if QIgG[total]>Qlim, with QIgG[spec]=IgGspec [CSF]/IgGspec[serum], and QIgG[total]=IgGtotal[CSF]/ IgGtotal[serum][1,3]. The upper reference range of QIgG[total], Qlim, was calculated according to Reiber's formula [1]. No lumbar puncture or phlebotomy was performed for this study, and no stored CSF or serum samples were used for this study. Instead, all data were retrieved retrospectively by an automated database search and analyzed anonymously. As clinical data were not available from all patients due to anonymization, a very conservative cut-off for AI positivity (2.0 instead of
1.3) was applied to preclude false-positive results. No other inclusion and exclusion criteria than OCB negativity and presence of a positive AI for any of those six viral and bacterial antigens in the same paired CSF/serum sample were applied. A positive AI was present for $\mathrm{Bb}$ in 7 cases (2.5-20.9; median, 4.794) for HSV in 4 cases (2.02-43.4; 2.28), for VZV in 7 (2-16.2; 2.42), for CMV in 1 (2.40), for MV in 1 (2.80), and for RV in 1 (2.10). In case \#1, HSV encephalitis was confirmed by PCR and a follow-up lumbar puncture (LP) confirmed the positive $\mathrm{AI}$, again in the absence of total-IgG OCB. In a second case with positive HSV-AI and negative total-IgG OCB, herpes simplex blisters in the area innervated by the second branch of the trigeminal nerve were present and the patient developed facial nerve palsy; the result was confirmed in a second CSF/serum samples obtained 8 days 
later. Confirmation from repeat lumbar puncture was also available in a case of neuroborreliosis (Table 1). All of these cases were associated with CSF pleocytosis. Other clinical diagnoses associated with positive viral AIs as provided by the senders included "encephalitis", "meningitis", "abducens and facial nerve paresis", and "clinically possible multiple sclerosis"; one VZV-AI positive patient was treated with tacrolimus (FK506), a potent immunosuppressant, at the time of LP (Table 1).

To assess the plausibility of the negative total-IgG OCB results, we analysed each patients' total-IgG CSF/ serum ratio (QIgG). Elevated QIgG in the absence of total-IgG OCB positivity would suggest a false-negative OCB result. However, QIgG was found to be normal in all patients (i.e. QIgG $<$ Qlim) as shown in Figure 1.

\section{Discussion}

These cases confirm that negative total-IgG OCBs in CSF samples might not always predict the absence of positive virus- or bacterium-specific IgG-AIs in patients with suspected CNS infection. Omitting AI testing in OCB negative patients might thus be a possible

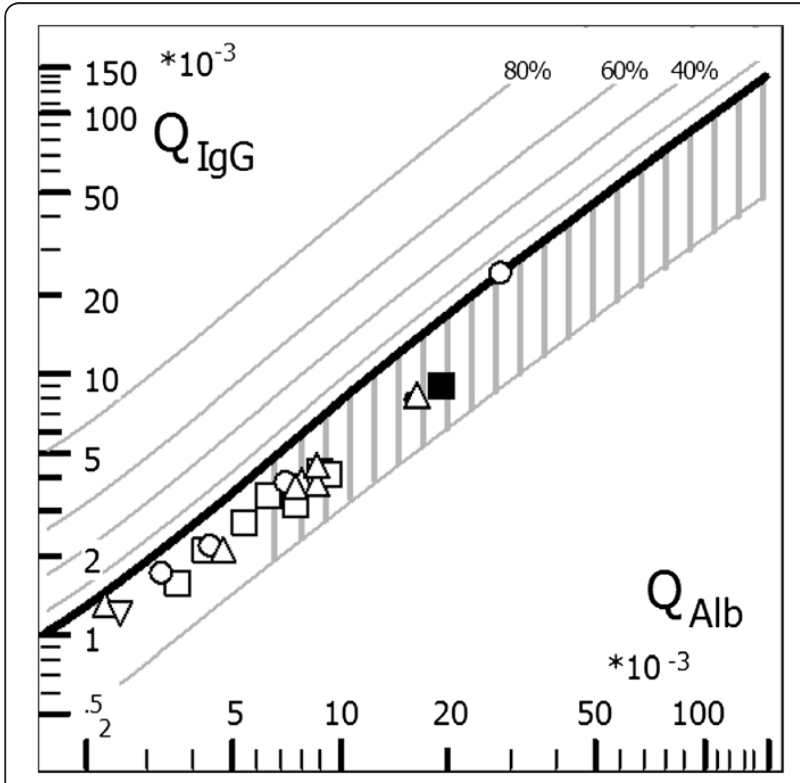

Figure $1 \mathrm{CSF} /$ serum quotient diagram for IgG ('Reibergram'). Individual CSF/serum ratios of lgG (QlgG) were plotted against CSF/ serum albumin ratios (QAlb). Values above the upper hyperbolic discrimination line Qlim indicate intrathecal total-lgG synthesis. Individual intrathecal fractions, IgIF, can be obtained by interpolation from the percentiles above Qlim. The Reibergram plot indicated that there was no intrathecal total-lgG synthesis in the patients tested here, confirming the negative $\mathrm{OCB}$ results. $\lg \mathrm{G}=$ immunoglobulin $\mathrm{G}$; QlgG = CSF/serum total-IgG ratio; QAlb = CSF/serum albumin ratio. Graph created using CSF Research Tool, Comed, Germany. Open squares: Borrelia burgdorferi, Bb; upward triangles: varicella zoster virus, VZV; open circles: herpes simplex virus, HSV; filled circles:

cytomegalovirus, CMV; filled squares: measles virus, MV; downward triangles: rubella virus, RV. diagnostic pitfall. In addition to VZV infection [2], positive AIs have previously also been reported in individual total-IgG OCB-negative patients with autoimmune disorders of the central nervous system including antiGAD antibody positive stiff person syndrome [4], anti-Ri positive paraneoplastic neurological syndromes (PNS) [5], anti-CV2/CRMP5 positive PNS [6], and anti-Yo positive PNS [3].

Around 2-5\% of patients with MS are negative for OCBs. This subgroup, which is small in relative numbers but not so small in absolute numbers due to the high prevalence of MS, is particularly challenging from a diagnostic point of view. A higher sensitivity of antigenspecific AI calculation compared to total-IgG OCB testing might therefore also be of potential relevance for the laboratory diagnosis of multiple sclerosis (MS), since a majority of patients with MS display a polyspecific humoral antibody response to a broad variety of viral and bacterial antigens as determined by AI calculation, with intrathecal antibodies to $\mathrm{MV}, \mathrm{RV}$ and $\mathrm{VZV}$ as its most common constituents (termed MRZ reaction or MRZR), as demonstrated by Reiber and others [7-9]. Apart from MS, a positive MRZR reaction has also been reported in rare cases of lupus and CNS involvement [7]. MRZR testing is currently performed by many CSF laboratories in Germany, but, in our experience, is so far almost exclusively done in OCB-positive patients. In line with the hypothesis that tests for antigen-specific IgG synthesis might be more sensitive than total-IgG OCB detection, Stich et al. recently reported on positive AIs for MV, RV, and VZV in 2/17 (12\%) total-IgG OCBnegative patients with MS but in none of 11 controls. Similarly, the authors found MV-, RV-, and VZVspecific bands in 3/17 (18 \%) total-IgG OCB-negative patients with MS as detected by affinity blotting using recombinant viral antigens and a highly sensitive chemiluminescence detection technique [8]. This is in accordance with a report by Frederiksen and Sindic (1998), who found CSF-restricted VZV- and mumps-specific bands in total-IgG OCB-negative patients with MS using a similar type of assay [9].

On the other hand, the fact that a subset of patients with MS presents with a monospecific AI elevation rather than the typical polyspecific, oligoclonal reaction, at least at first presentation [10], and that the spectrum of positive AIs can be broader than just MV, RV, and VZV and include (though less frequently) positive AIs for $\mathrm{Bb}$, toxoplasma and other viral and bacterial agents can pose differential diagnostic challenges in some cases. Accordingly, in patients in whom MS is a reasonable differential diagnosis, AIs for at least the three main constituents of the MRZ reaction ( $M V, R V$, and VZV) should be determined (approximate costs in Germany, $\sim 20-30$ 
$€ /$ AI determination). Moreover, AI results - as laboratory results in general - must always be interpreted in the context of the patient's overall clinical and neuroradiological presentation and the patient's CSF findings such as white cell count and differentiation, CSF/serum total-IgM ratios or total-IgA ratios before a definite diagnosis can be made [1,11]. BbIgM assessment can be useful in patients with suspected acute $\mathrm{Bb}$ infection; however, it was negative in 6/6 patients in our study (not determined in one).

The reason for the higher sensitivity of AI detection compared to total-IgG detection in the patients reported here is unknown. Infections are often associated with severe blood-CSF barrier dysfunction; the presence of OCB may become undetectable in those cases because of a relative increase of the bloodderived polyclonal IgG fraction. In such cases OCBs may possibly become positive after normalization of barrier dysfunction. However, high CSF/serum albumin ratios were present only in a subset of our patients (see Figure 1). Moreover, the higher sensitivity of antigen-specific OCBs compared to total-IgG OCBs detection as demonstrated in a number of studies [3-5] argues in favour of additional, so far unidentified factors specific for total-IgG OCB detection rather than OCB detection in general.

Possibly, AIs might become positive earlier than totalIgG OCBs. If so, AI assessment might be particularly useful at disease onset. However, as OCBs remained negative in the few patients with follow-up LPs in our study, our study provides no direct evidence for this hypothesis.

There are some caveats. First, as it is the case with IgG responses in general, there might be a delay in AIs becoming positive as indicated by case 1 in Table 1 ; this patients became positive for HSV only at lumbar punctures (LP) \#2 and LP \#3. However, similar problems apply to HSV PCR, which is sometimes negative at first LP and becomes positive only with time. Therefore, repeat LP is recommended if the initial LP is negative. Second, an expansion of the polyspecific, intrathecal immune response in MS over time has been described [10]. Accordingly, repeat LP might possibly be useful also in individual patients with suspected MS but missing MRZR reaction at first LP. Third, cross-reactivity between VZV and HSV is a well-known phenomenon. However, the true-positive AI generally yields higher values, which usually allows identifying the relevant agent (see patient 1, LP \#3). It is therefore recommended to test always for both VZV-AI and HSV-AI. Third, the 21 cases presented here were collected over a period of 9 years, suggesting that the phenomenon is relatively rare; however, it is potentially of high relevance for the individual patient.
In summary, the cases reported here indicate that AI testing should not generally be omitted in total-IgG OCB-negative patients and provides a rationale for systematic and prospective studies on the comparative sensitivity and specificity of AI and total-IgG OCB testing in infectious diseases of the CNS as well as in other indications.

\section{Abbreviations}

Al: antibody index; Bb: Borrelia burgdorferi; CSF: cerebrospinal fluid; CMV: cytomegalovirus; CNS: central nervous system; HSV: herpes simplex virus; IgG: immunoglobulin G; LP: lumbar puncture; MRZR: measles virus, rubella virus, and varicella zoster virus reaction; MS: multiple sclerosis; MV: measles virus; NB: neuroborreliosis; OCB: oligoclonal lgG bands; PCR: polymerase chain reaction; PNS: paraneoplastic neurological syndromes; Q: ratio; RV: rubella virus; VZV: varicella zoster virus; WCC: white cell count.

\section{Competing interests}

The authors declare that they have no competing interests.

\section{Acknowledgements}

This work was supported by research grants from Bayer Healthcare and MerckSerono to BW. We are grateful to D. Menzel, R. Herbst, M. Hoehne, and H. Pahl, Department of Clinical Chemistry, Ludwig Maximilian University, Munich, Germany, for excellent technical assistance.

\section{Author details}

${ }^{1}$ Division of Molecular Neuroimmunology, Department of Neurology, University of Heidelberg, Im Neuenheimer Feld 400, 69120 Heidelberg, Germany. ${ }^{2}$ Department of Clinical Chemistry, Klinikum Grosshadern, Ludwig Maximilian University, Munich, Germany.

\section{Authors' contributions}

S.J. conceived the study, analysed the data, and wrote the initial draft; P.E. and M.W. conducted and interpreted the laboratory tests; S.J., P.E., B.W., and M.E. revised the manuscript for important intellectual content. All authors have read and approved the final version of the manuscript.

Received: 14 April 2012 Accepted: 11 July 2012

Published: 31 July 2012

\section{References}

1. Reiber $\mathrm{H}$ : Cerebrospinal fluid-physiology, analysis and interpretation of protein patterns for diagnosis of neurological diseases. Mult Scler 1998, 4:99-107.

2. Felgenhauer $\mathrm{K}$, Reiber $\mathrm{H}$ : The diagnostic significance of antibody specificity indices in multiple sclerosis and herpes virus induced diseases of the nervous system. Clin Investig 1992, 70:28-37.

3. Stich O, Graus F, Rasiah C, Rauer S: Qualitative evidence of anti-Yo-specific intrathecal antibody synthesis in patients with paraneoplastic cerebellar degeneration. J Neuroimmunol 2003, 141:165-169.

4. Jarius S, Stich O, Speck J, Rasiah C, Wildemann B, Meinck HM, Rauer S: Qualitative and quantitative evidence of anti-glutamic acid decarboxylase-specific intrathecal antibody synthesis in patients with stiff person syndrome. J Neuroimmunol 2010, 229:219-224.

5. Jarius S, Stich O, Rasiah C, Voltz R, Rauer S: Qualitative evidence of Ri specific lgG-synthesis in the cerebrospinal fluid from patients with paraneoplastic neurological syndromes. J Neurol Sci 2008, 268:65-68.

6. Stich O, Rauer S: Antigen-specific oligoclonal bands in cerebrospinal fluid and serum from patients with anti-amphiphysin- and anti-CV2/CRMP5 associated paraneoplastic neurological syndromes. Eur J Neurol 2007, 14:650-653.

7. Graef IT, Henze T, Reiber H: Polyspecific immune reaction in the central nervous system in autoimmune diseases with CNS involvement. Z Arztl Fortbild (Jena) 1994, 88:587-591.

8. Stich O, Kluge J, Speck J, Rauer S: Detection of virus-specific (measles, rubella, zoster) oligoclonal lgG-bands in CSF from multiple sclerosis patients without oligoclonal bands of total IgG. Mult Scler 2009, 15:S86. 
9. Frederiksen JL, Sindic CJ: Intrathecal synthesis of virus-specific oligoclonal $\mathrm{lgG}$, and of free kappa and free lambda oligoclonal bands in acute monosymptomatic optic neuritis. Comparison with brain MRI. Mult Scler 1998, 4:22-26.

10. Petereit HF, Reske D: Expansion of antibody reactivity in the cerebrospinal fluid of multiple sclerosis patients - follow-up and clinical implications. Cerebrospinal Fluid Res 2005, 2:3.

11. Reiber H, Peter JB: Cerebrospinal fluid analysis: disease-related data patterns and evaluation programs. J Neurol Sci 2001, 184:101-122.

doi:10.1186/2045-8118-9-14

Cite this article as: Jarius et al:: Usefulness of antibody index assessment in cerebrospinal fluid from patients negative for total-IgG oligoclonal bands. Fluids and Barriers of the CNS 2012 9:14.

\section{Submit your next manuscript to BioMed Central and take full advantage of:}

- Convenient online submission

- Thorough peer review

- No space constraints or color figure charges

- Immediate publication on acceptance

- Inclusion in PubMed, CAS, Scopus and Google Scholar

- Research which is freely available for redistribution 